\title{
Exploration and Analysis of Exercises of Subjective Induction Sketch from Nature in Design Color Teaching
}

\author{
Xiaofei Zhang \\ Arts and Design School of Huanghe Science and Technology College \\ Zhengzhou, Henan, 450000 \\ e-mail:359163743@qq.com
}

\begin{abstract}
To sketch colors is a precondition for realizing functions of colors while it is also a shortcut to train designers' observational ability, aesthetic judgment and creative power. Design-oriented inductive sketch from nature will realize the shaping ability of decorative colors. Subjective induction color sketch is a kind of exercises conducted on basis of realistic induction subjects. It is a way of expression that features more of decoration language and decorative characters and must be grasped from aspects of composition, shaping, and colors, etc.
\end{abstract}

\section{Keywords-Design Color; Inductive Sketch; Subjectiveness}

\section{INTRODUCTION}

Design colors are a professional basic curriculum most new college students majoring in arts and design will learn at the beginning of their college life. Mission of the curriculum is to help student transit from their painting-oriented thought to design-orientated thought. How to make basic design curricula serve better professional curricula and how to help freshman to face squarely with their professional learning are focuses of reform and practice of design color curricula. And subjective induction sketch of objective images is the key of the teaching system of design color curricula.

\section{Change of Design Color Thought}

From general color sketch from nature to design-oriented color sketch a series of transitions will happen.

Firstly, on aspect of creative conception, painting colors stress spirituality, emotionality and appreciativeness and express the inner feelings of the painter; while design colors lead by creative concepts emphasize functions and roles, and must consider the customer's aesthetic requirements and environmental conditions.

Secondly, on aspect of observation methods, painting colors will observe, analyze and express at fixed point of view and time the relationship between the object and the light source colors and ambient colors as well as the law of change of light and color; while design colors emphasize summarizing and refining colors, therefore, they will not be bound by time and space and give priority to expressing inner feelings and physiological visual perceptions.

Thirdly, on aspect of expression approaches, painting colors must depict the image according to its form and color, give a truthful expression to the form, structure, color and texture of the object, show spatial relationship between the full and the empty and give three-dimensional effects, therefore, they will render an objective recording of natural colors; while design colors emphasize juxtaposition, change, combination and configuration of colors (patches) to highlight decorativeness of colors.

\section{CONTENT OF EXERCiSES OF SUBJeCtive InduCtion COLOR SKETCH}

Subjective induction sketch changes the all-inclusive observation and expression habit by weakening volume and colors in objective reality and emphasizing and expressing subjective changes of forms and colors so as to highlight synergy of forms and colors and help understand the overall plane effects. This style is widely used and frequently seen from traditional arts to modern arts, from oriental arts to occidental arts, and from modern painting to modern design.

\section{A. Composition Exercise}

Mr. Zhang Yanyuan, an artist from Tang Dynasty, once spoke highly of the role of composition. He referred to composition as the overriding element in painting, which dominates the form and color. Therefore, before using the brush to paint, we must demand our craftsmanship and organize carefully. Only by doing this, can we achieve the best results by give full play to the role of composition.

Subjective induction sketch stresses complanation during composition. It will change artificially the order of objective existence according to the design concept and cavalier perspective and multi-point perspective are most effective in realizing complanation.

Cavalier perspective is the traditional method adopted by Chinese painters. Its points of view flow from one to another, 
like a person riding on a helicopter to shoot scenes on his way on the picture.

Multi-point perspective is freer than cavalier perspective in that as long as the organization needs it, the point of view will be moved at will. By cavalier perspective or random perspective, the painting will show three-dimensional effects and spatial effect, however, the image in the painting will shrink in size. When drawing, we can combine three-dimensional composition with eye-level composition to enrich the painting and facilitate arrangement of objects in the painting according to their significance.

\section{1) Eye-level Composition}

Eye-level composition breaks the law of perspective. This composition has no fixed points of view when observing the image, it adopts mobile line of sight and parallel perspective. Objects far or near will be arranged on the same plane. Generally, objects at the depth of the natural view will be moved sideways on the painting. The line of sight is perpendicular to the facade of the image and there is no depiction of the top surface and side surfaces. To achieve the above complanation effects, in the natural order of objects must be firstly changed to the artificial order before they are put on the painting. This demands subjective arrangement of colors and forms in the painting. Ancient works like the stone relief Hauling Three-legged Bronze Vessel from Sishui River, the Picture of Water and Land Battleship and Banquet Music Playing Engraved on Bronze Kettle of the Warring Period and Painted Pattern of Silvering and Gold-plating on Bronze Chariots of Han Dynasty, all show their content on a pane and is not bound by time and space. There is no overlapping between parts of an image or between images. They are all shown in silhouette and by interlacing together in a delicate way to present all characters of the image on the painting. This composition makes the form less complex and shows orderly beauty. Therefore, the composition with strong summarizing ability and a language of simple and clear images give priority to decorative effects.

Chinese traditional paper-cutting, Greek terra cotta vases and Babylonian frescos all adopt eye-level composition. It looks like you spread the flowers and grass even and pressed them flat to make them herbarium. This composition intentionally pursues artificial complanation by removing three-dimensional effect and the spatial depth between different images, so as to make every image above the eye line.

\section{2) Three-dimensional Composition}

This composition adopts cavalier perspective and random perspective on basis of eye-level composition. This is done by setting up an elevation and drawing from its apexes parallel lines with an angle of 45 degrees to its left and right to form the top and sides of a three-dimensional image. Typical examples of this composition include A Riverside Scene at Qingming Festival by Zhang Zeduan, an artist of Northern Song Dynasty and pictures on portrait bricks.

While doing composition at sketching nature, we can move the object from left to right or from top to bottom on the picture while we will abandon some unnecessary details. The most important thing in composition is to arrange in the picture images of different objects according to some order, so as to make the painting an organic entity complete in form and uniform in crafts.

\section{B. Configuration Exercise}

\section{1) Complanation}

Complanation is an important and basic to decorative shaping. Compalnation is to change three-dimensional images into two-dimensional graphic images, namely arranging images only in the scope of length and width by adopting eye-level perspective.

Due fronts and due flanks refer to the only front or flank that can be seen by adopting eye-level observation when the visual angle is parallel to the image at looking at its front or flank. The overhead views and upward views refer to the only top or bottom that can be seen when looking downward or upward perpendicularly to the image. The unfolded side refers to the invisible side of the image that is depicted because of requirements of the picture. This makes the picture more interesting. Reversal and rotation from left to right or top to bottom used in folk animal pictures of China and the methods used by cubist Picasso in expressing depicting human face and body all belong to treatment approach of unfolded side. Silhouette method shows the outline of an image. It discards the visible texture, structure, undulation and layers of the image and only gives emphasis to outer shape of the image so as to make the picture simpler and purer.

Arrangement of images should be decided by their spatial positions on the picture with eye-level perspective instead of their actual positions in real world. Compared with objective induction approach, this way is much more subjective and freer.

When doing subjective induction sketches, people must design and think carefully before start painting; they cannot start painting at will as they do at drawing ordinary paintings by applying rough colors first, then perfecting and finishing the painting step by step. People must study and deliberate carefully over the composition, shapes and colors before they start drawing and applying colors. Strict and detailed line drawing drafts must be drawn to lay a good foundation for depicting the shape and color in a later stage.

\section{2) Deformation}

Subjective induction sketches adopt cavalier perspective in aspect of composition. They emphasize complanation, therefore, abandon difference of depths of objects and focus more on changes of the form. If we do not alter the objective form and only put the simplified plane form into the painting, we will see an discordant and inharmonious picture. Deformation is sublimation of the natural objective forms, it is insight and imagination. Deformation methods often used are as follows:

a) Simplifying and Outlining: Simplification is to outline and refine natural forms by removing those unnecessary and uncharacteristic trifles. The process of 
simplification is a process of distilling the natural images, which needs to extract and retain typical features from complex shallow facets of the images by cutting off some trifles to highlight the wholeness and make the image purer and clearer.

b) Exaggeration: Exaggeration is an important method in artistic creation. It will surprise people extraordinary visual effects. Exaggeration is not boundless and aimless. It is used to strengthen typical features of images being depicted with a view to highlighting image of the objects and enhance artistic appeal.

Proportional Exaggeration. There are two kinds of proportional exaggerations, one is extended deformation wile the other is contracted deformation. By extended deformation, image of the object will become more slender and make people feel it is light, tall and straight. Contracted deformation will reduce length of the object, render it wider and fatter and make people feel it is heavy, awkward and simple.

Perspective Exaggeration. Using perspective principles to do exaggeration will give people funny feelings about orientation, space and movements. Perspective exaggeration includes different distortion angles like front, sideways, downward and upward, etc. It will produce weird visual effects by deforming the picture.

Partial Exaggeration. In order to achieve a special aim, one part of the people may be specifically emphasized in spite of the other parts so as to highlight the theme or enhance its appeal. For example, when deformation happens on a human image, part of his body such as eye, nose, mouth, head, limbs or torso may be highlighted according to different needs. In commercial arts, it is often seen that partial exaggeration is used to underline the theme or communicate the information.

Overall Exaggeration. Overall exaggeration renders the form with some proclivity, for example, the fat becomes fatter, the thin becomes thinner, the long becomes longer and the short becomes shorter. The overall image features can give people distinctive visual feelings, for example, portrait stones of China, stone engravings of Maya and wooden sculpture of Africa all give people a sense of power, simplicity and awkwardness. However, vase paintings of Greece, human portraits by Beardsley and Matisse give people a sense of grace and easiness.

Conformal Exaggeration. Conformal exaggeration pursues changes with constraints. Changes and arrangement of the image must adapt to the some specific space and shapes. This deformation with constraints from specific space and structure is called conformal exaggeration. When it comes to shaping Conformal exaggeration does not work on structure of the object but on the space that the object must adapt to. However, this exaggeration is not adapting the object mechanically to the space. Under usual circumstances, the space is compatible with the exaggerated form. The space and the form rely on each other to become an organic entity.

c) Enrichment by Adding Elements: Addition must be done according to requirements of the content and form. For example, some textures can be added to the image to make diversified, less monotonous and more interesting.
Addition of Texture:If the conception and composition require, texture can be added to ornament a dull image and render it with reasonably alternate density and sparseness, thus it looks more vivid, harmonious and rhythmical. When adding texture, we can associate with the theme of the painting. And we can use some meaningful and connotational texture to impute new meaning and significance into the image.

Additions Needed by the Shape: Points, lines, facets or other elements can be added in the image because of requirements of the shape. This method will make the painting richer and fuller while it also enhances the decorative effect and beauty of shape of the painting. It is commonly used in folk arts and crafts.

\section{Color Designing Exercise}

At color designing, changes will be done on basis of colors of the object. We will not do simple emulation of proper colors, ambient colors and conditional colors. Instead, subjective treatment will be added after showing respect to and recognizing basic colors of the object. When it comes to method of expression, even application of color patches is often used to present the contrast between forms and color patches, so as to render the painting with strong visual effects of complanation and decoration. The following aspects must be considered when doing color design:

1) Compliance with Proper Colors: Natural images boast various relations between colors, which we can give reference to at color designing and base our association and subjective creation on. Proper colors of different images are still the primary colors used at presenting.

2) Highlighting Subjective Color Change: Because the author has different thoughts and aspiration, his subjective colors can be highlighted at color designing to help representation. Subjective color change needs to consider the following aspects: Color limiting: for example, the non-color line consisting of black, white and grey; or color lines of three colors or five colors.

Emphasizing subjective color tone: cold colors, warm colors, neutral colors, high colors and low colors.

Emphasizing color contrast. For example, extreme contrast between white color and black color, contrast in color, brightness, purity and property, or contrast between bordering colors, contrasting colors, and complementary colors.

3) Pursue Harmony among Colors: Color induction adheres to harmony as a principle in painting representation. Essence of induction is orderliness and clearness. Of course, the harmony mentioned here should be understood in a narrow sense. The harmony means a unified as well as opposite relationship, or rather a unity of diversity. Thus, will produce the ever-changing but unified effects in the painting.

Multiple practices in teaching design color classes show that subjective induction sketch exercise plays an important role helping student to change from their thought of color under exam-oriented education to thought of design color in professional college education and help improve their aesthetic judgment and creative power. 


\section{ACKNOWLEDGMENT}

[Foundation Project] The project of "Experimental Zone for Innovation of Arts and Design Talent Cultivation Mode" established in Huanghe Science and Technology College Experimental Zone for Innovation of Talent Cultivation Mode in 2011

\section{REFERENCES}

[1] Zhang Ruhua, Zhang Jiaming, Gu Chen, et al. Design Color [M]. Beijing: China Youth Press, 2010.

[2] Lu Qi, From Color to Design [M]. Hangzhou: Publishing House of China Academy of Arts, 2004. 